\title{
Variação temporal e espacial na dieta de Trachelyopterus galeatus (Siluriformes, Auchenipteridae) em dois reservatórios no semiárido Neotropical
}

\author{
José I. M. Sousa', Jean C. D. Oliveira², Jônnata F. Oliveira², \\ Luzia G. F. Rebouças' , José L. C. Novaes², Rodrigo S. Costa² \& Danielle Peretti'
}

\begin{abstract}
1. Laboratório de Ictiologia, Departamento de Ciências Biológicas, Universidade do Estado do Rio Grande do Norte, Av. Prof. Antônio Campos, Costa e Silva, 59625-620 Mossoró, RN, Brasil. (danielleperetti@uern.br).

2. Laboratório de Ecologia de Peixes e Pesca Continental, Departamento de Ciências Animais, Universidade Federal Rural do Semiárido, BR 110 - Km 47, Costa e Silva, 59625-900 Mossoró, RN, Brasil.
\end{abstract}

Received 24 October 2016

Accepted 06 September 2017

DOI: $10.1590 / 1678-4766 e 2017040$

\begin{abstract}
Temporal and spatial variation in diet Trachelyopterus galeatus (Siluriformes, Auchenipteridae) in two reservoirs in the semi-arid Neotropical. Fish feeding provides data for discussion of theoretical aspects serving purposes as the basic knowledge of the biology of a species by understanding the trophic organization in each environment. The objective was to analyze the food composition and morphology associated with the eating habits of Trachelyopterus galeatus (Linnaeus, 1766) and evaluates the dietary changes over time. The collections of individuals took place on a quarterly basis, during the months of February, May, August and November 2013, through gillnets with different meshes distributed in eight points in the Santa Cruz reservoir and five points in the Umari reservoir, both located in the Rio Grande do Norte. From the items identified in diets were obtained the frequencies of occurrence and volumetric, combined in the calculation of Alimentary Index (IAi). The volumes of the items were ordered in NMDS and their differences tested by PERMANOVA. The contribution of each food item and differences between reservoirs was assessed by SIMPER analysis. The analysis of stomach contents allowed the classification of the species as carnivorous, noting there are significant temporal changes in the species diet, with the morphological characteristics according to the consumption of food items.
\end{abstract}

KEYWORDS. Diet, trophic organization, fish, temporal variation.

RESUMO. Alimentação de peixes propicia dados para discussão de aspectos teóricos atendendo a propósitos como o conhecimento básico da biologia de uma determinada espécie através do entendimento da organização trófica em um determinado ambiente. Assim, objetivou-se analisar a composição alimentar e a morfologia, associadas ao hábito alimentar de Trachelyopterus galeatus (Linnaeus, 1766) e avaliar as alterações da dieta ao longo do tempo. As coletas dos indivíduos ocorreram trimestralmente, durante os meses de fevereiro, maio, agosto e novembro de 2013, por meio de redes de esperas com diferentes malhas distribuídas em oito pontos no reservatório de Santa Cruz e em cinco pontos no reservatório de Umari, ambos localizados no Rio Grande do Norte. Dos itens identificados da dieta foram obtidas as frequências de ocorrência e volumétrica, combinadas no cálculo de Índice Alimentar (IAi). Os volumes dos itens foram ordenados em NMDS e as suas diferenças testadas em PERMANOVA, a contribuição de cada item alimentar para as diferenças entre os reservatórios foi avaliada através da análise SIMPER. As análises dos conteúdos alimentares permitiram a classificação da espécie como carnívora, constatando-se significativas alterações temporais em sua dieta, com as características morfológicas em conformidade ao consumo dos itens alimentares.

PALAVRAS-CHAVE. Dieta, organização trófica, peixe, variação temporal.

Alimentação é um dos mais importantes aspectos da biologia dos peixes, que interfere diretamente na estrutura e composição de populações (PESSOA et al., 2012). A compreensão da utilização diferencial dos recursos dos ecossistemas, denominada partilha de recursos, é essencial na investigação das assembleias de peixes neotropicais. Esse conhecimento possibilita entender as interações que existem entre as espécies, como também determinar como as guildas tróficas estão distribuídas ao longo do tempo (SILVA et al., 2012), uma vez que a maioria das espécies de peixes de água doce dispõe de uma ampla gama de estratégias e táticas alimentares, consumindo grande número de itens, favorecendo adaptações às novas condições impostas pelo ambiente (HAHN \& FUGI, 2007).

Em ambientes represados, alterações na dieta de peixes podem ser dirigidas por modificações espaço-temporais, que apresentam grandes variações no regime hidrológico, causando modificações na oferta de recursos alimentares do ambiente (NovAKOWSKI et al., 2007). Outros fatores são o oportunismo trófico, por substituir itens escassos por outros abundantes de acordo com a disponibilidade do recurso alimentar (DAVIES et al., 2008). No entanto, para a aquisição de alimento é necessário que os peixes disponham de mecanismos apropriados para utilizá-los e aproveitá-los. Isso 
inclui adaptações morfológicas, principalmente a morfologia da boca e do aparelho digestório, que potencializem as funções alimentares permitindo a exploração de diversos estratos do corpo d'água em busca do alimento (PeretTi \& ANDRIAN, 2008).

Eventos de perturbações no ambiente têm efeito sobre a abundância de espécies (Oliverra et al., 2011), sendo que a continuidade deste distúrbio pode eliminar aquelas mais sensíveis (FeliPE \& SUAREZ, 2010). As secas são perturbações de grande escala que afetam os ecossistemas de água doce em todo o mundo, sendo bastante estudadas em diferentes regiões, devido a sua importância no ciclo hidrológico e na manutenção dos organismos (CosTA et al., 2016). A seca causa diminuição no nível de água nos reservatórios provocando mudanças nas condições ambientais e na comunidade (Medeiros et al., 2014), sendo os peixes o principal grupo afetado (PINTo et al., 2011). Como observado na região semiárida do Brasil, onde a baixa precipitação pluviométrica nos últimos anos afeta as populações de peixes, em especial os reofílicos que dependem das cheias para realizar a migração (Araúso et al., 2016), e a estrutura trófica do ambiente (Oliveira et al., 2016a,b).

Trachelyopterus galeatus Linnaeus, 1766 (Siluriformes, Auchenipteridae) é conhecida popularmente no semiárido como Cangati, e em outras regiões como anujá, cachorrinho, cachorro do padre, cabeça de ferro e cumbá ronca-ronca (BorGEs et al., 1999). Alimenta-se principalmente de peixes, detrito, artrópodes, vermes e de frutas (MÉRONA \& RANKINDE-MÉrona, 2004), o que faz da espécie um importante elo na transferência de energia do sistema terrestre para o sistema aquático. Apresenta hábito noturno (NAKATANI et al., 2001) e pode sobreviver em ambientes com baixas concentrações de oxigênio (SoARes et al., 2006).

Considerando os efeitos da baixa precipitação pluviométrica nos reservatórios e como esta interage com as espécies de peixes, objetivou-se analisar a composição alimentar e morfologia associada ao hábito alimentar de Trachelyopterus galeatus e verificar, temporalmente, a variação na dieta da espécie presente nos reservatórios de Santa Cruz e Umari, Rio Grande do Norte. As hipóteses testadas foram: (i) a espécie altera a alimentação devido às alterações temporais, como a precipitação e espacial (entre os reservatórios) e (ii) a espécie é generalista, sendo a sua dieta confirmada pela morfologia do trato alimentar.

\section{MATERIAL E MÉTODOS}

Área de estudo. Na Bacia do rio Apodi-Mossoró, segunda maior do Rio Grande do Norte, estão registrados 22 reservatórios, todos com a finalidade de abastecimento humano e uso na agropecuária, dentre os quais se destacam os de Santa Cruz e Umari (SEMARH, 2016). O reservatório Santa Cruz, inaugurado no ano de 2002, está localizado no município de Apodi (0546'13.27”'S 3748’33.01”W), é formado pelo barramento do rio Apodi/Mossoró com uma bacia hidrográfica de $4.264,00 \mathrm{~km}^{2}$ e possui a capacidade de armazenar até 600 milhões de $\mathrm{m}^{3}$ de água, sendo o segundo maior reservatório do RN (ANA, 2007). O reservatório Umari, também inaugurado em 2002, está localizado no município de Upanema (0542’04.24”S 37¹4’34.28”W), é formado pelo barramento do rio do Carmo e possui uma bacia hidrográfica de $1.533 \mathrm{~km}^{2}$, com capacidade de armazenamento de até 300 milhões de $\mathrm{m}^{3}$ de água, sendo o terceiro maior do RN (ANA, 2007).

Na região semiárida, a temperatura média anual é de $28,5^{\circ} \mathrm{C}$, e precipitação média anual de $772 \mathrm{~mm}$, registrandose uma distribuição irregular durante o ano (ANA, 2007). Para a caracterização do regime pluviométrico durante o estudo foram utilizados os dados de precipitação pluviométrica para as cidades de Upanema e Apodi fornecidos pela Empresa de Pesquisa Agropecuária do Rio Grande do Norte - EMPARN. A precipitação em ambos os reservatórios variou em 2013, onde os dois primeiros períodos (fevereiro e maio) apresentaram o maior acúmulo de precipitação em relação aos dois últimos (agosto e novembro) no reservatório de Umari, e maior precipitação em maio no reservatório de Santa Cruz, não havendo registro de chuvas no mês de agosto em ambos (Fig. 1).

Amostragens. As capturas dos peixes ocorreram trimestralmente, durante os meses de fevereiro, maio, agosto e novembro de 2013. O esforço foi padronizado através da utilização de 11 redes de espera por ponto de coleta, com malhas de $12 ; 15 ; 20 ; 25 ; 30 ; 35 ; 40 ; 45 ; 50 ; 60$ e 70 $\mathrm{mm}$ (entre nós adjacentes), com $15 \mathrm{~m}$ de comprimento e altura entre $1,8 \mathrm{~m}$ e $2,0 \mathrm{~m}$, totalizando uma área de rede de $301,8 \mathrm{~m}^{2}$. Os aparatos de pesca foram instalados ao entardecer (17h) em pontos paralelamente às margens dos reservatórios, permanecendo expostas por um período de $12 \mathrm{~h}$. No reservatório de Santa Cruz as coletas ocorreram em oito pontos distribuídos ao longo do ambiente (Fig. 2A); em Umari, por se tratar de um reservatório de menor porte, as coletas ocorreram em cinco pontos (Fig. 2B).

Procedimentos com os espécimes coletados. Após captura, os exemplares foram transferidos para laboratório, triados e identificados, onde passaram por biometria e retirada dos estômagos, os quais foram mantidos em solução de formalina a $10 \%$ (durante um período de $72 \mathrm{~h}$ ) e, posteriormente, retirados e conservados em álcool $70 \%$ até a análise da dieta. Alguns espécimes foram fixados e enviados à Universidade Federal da Paraíba - UFPB, onde a identificação foi confirmada por taxonomista, e depositados na coleção ictiológica dessa instituição (catálogo UFPB: 8961).

Análise dos conteúdo estomacal. Os itens alimentares foram identificados, sob microscópio estereoscópico e óptico, ao menor nível taxonômico possível, com auxílio de literatura especializada (MCCAFFERTY, 1981; NeEDHAM \& NeEDHAM, 1982; Merritt \& Cummins, 1996). Dos itens identificados, foi obtido o volume, seguindo doi procedimentos de acordo com o tipo e dimensão do alimento: i) através de provetas graduadas, cujo volume é dado pelo deslocamento de líquido (Hynes, 1950; Hyslop, 1980; Zavala-Camin, 1996), e ii) pelo método de placa de Petri milimetrada (Hellawell \& ABEL, 1971). Neste método, os itens são separados de 


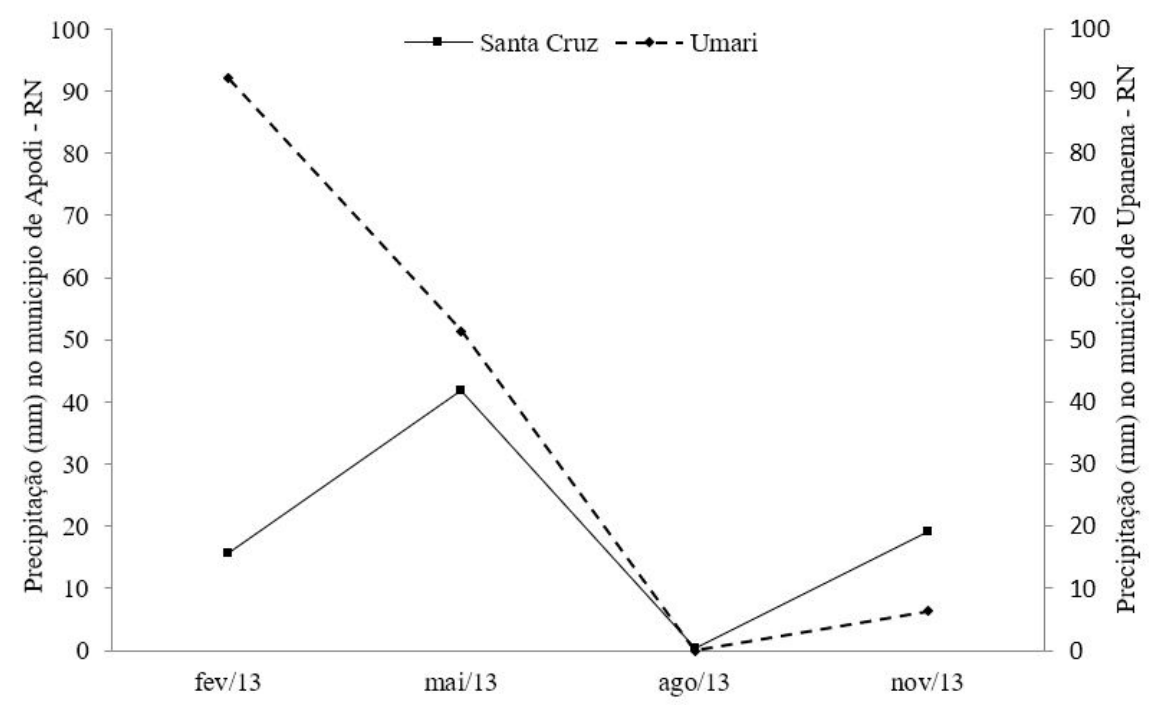

Fig. 1. Precipitação pluviométrica acumulada nos meses de fevereiro, maio, agosto e novembro de 2013, no reservatório de Santa Cruz, município de Apodi, e no reservatório de Umari, município de Upanema, Rio Grande do Norte, Brasil (Fonte dos dados: EMPARN, 2016).

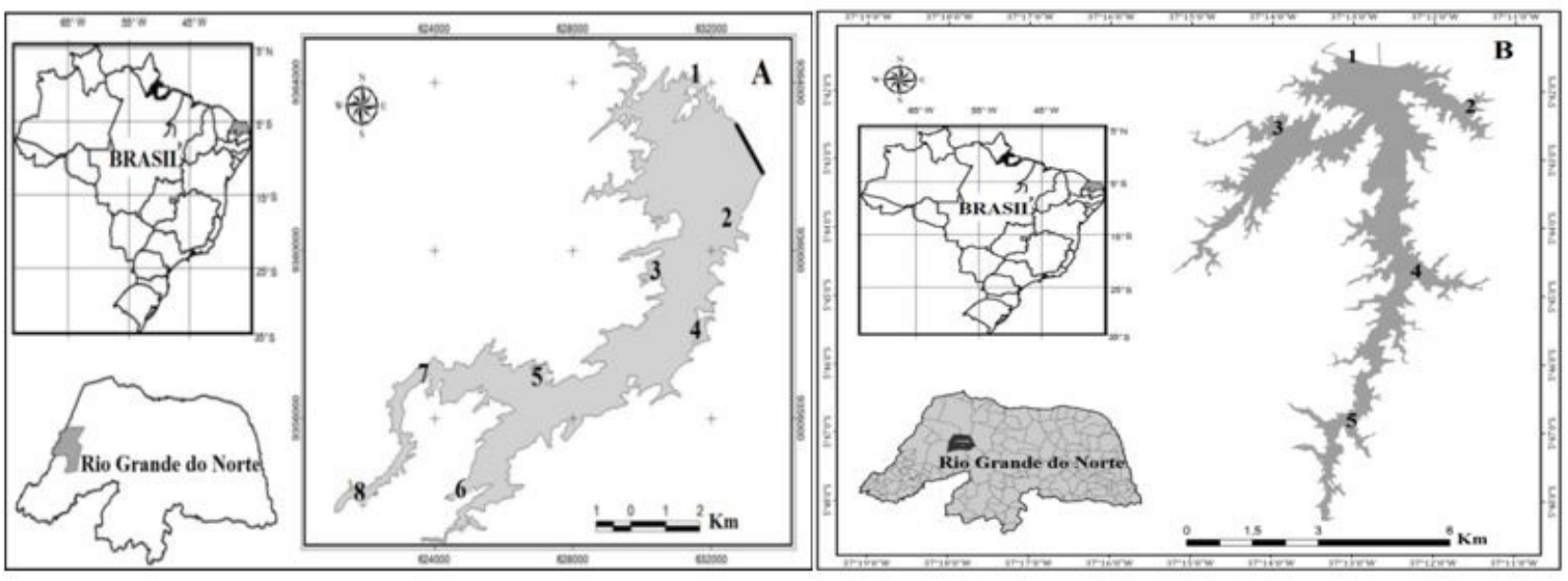

Fig. 2. Localização dos pontos de coletas, nos reservatórios de Santa Cruz (A) e de Umarí (B), Rio Grande do Norte, Brasil nos períodos de fevereiro, maio, agosto e novembro de 2013.

acordo com o menor nível taxonômico, e espalhados sobre uma placa de Petri com alturas padronizadas em $1 \mathrm{~mm}^{3}$. Os itens são compactados contra lâminas de vidro de $1 \mathrm{~mm}$ de espessura, pelas laterais e por cima, formando blocos de $1 \mathrm{~mm}^{3}$, os quais têm seu volume mensurado em $\mathrm{mL}$ (BASTOS et al., 2013).

Dos dados da dieta foram obtidas as frequências de ocorrência (Fo, expressa o número de estômagos que contém um determinado item em relação ao total de estômagos analisados) e volumétrica [Fv, expressa a contribuição do volume (em $\mathrm{mL}$ ) de cada categoria em relação ao total de todos os conteúdos analisados] (HyNes, 1950; HysLop, 1980; Rosecchi \& Nouaze, 1987; Zavala-Camin, 1996), cuja associação das frequências dá origem ao cálculo do Índice Alimentar (IAi), que utiliza simultaneamente os métodos de frequência de ocorrência e volumétrico, gerando um índice que evidencia os principais recursos da dieta (KAWAKAMI \& VAZZOLER, 1980).
Para avaliar os itens preferenciais da alimentação e determinar o hábito alimentar da espécie, foi utilizado a escala proposta por RoseCCHI \& NOUAZE (1987), sendo: IAi $>50 \%$ - item preferencial; $25<\mathrm{IAi}<50 \%$ - item secundário; IAi $<$ $25 \%$ - item acessório. De acordo com os itens preferenciais, a espécie foi enquadrada na devida categoria trófica.

Análise estatística. Os valores de IAi da espécie foram utilizados para determinar os itens preferenciais e secundários da dieta. Com intuito de avaliar a utilização dos recursos alimentares pela espécie ao longo do período de amostragem (por reservatório), foram utilizados os volumes dos itens alimentares numa matriz de similaridade, usando o coeficiente de similaridade de Bray-Curtis. O resultado de cada procedimento foi utilizado em Análises de Ordenação de Escalonamento Multidimensional Não Métrico (NMDS), e as diferenças na alimentação testadas por meio de Análises de Variância Multivariada Permutacional (PERMANOVA), utilizando o índice de similaridade de Bray-Curtis e os meses 
de coletas comparados pelo teste post-hoc da PERMANOVA. A contribuição de cada item alimentar para as diferenças observadas entre os reservatórios foi avaliada através da análise de SIMPER. Para as análises dos dados foi utilizado o programa estatístico PAleontological STatistics (HAMMER, 2017).

Procedimentos das ilustrações do trato digestório. O estudo da morfologia do trato alimentar foi conduzido com 15 exemplares adultos, baseando-se no grau de maturação sexual de acordo com a escala de VAzzoLER (1996). As observações foram feitas em indivíduos fixados em solução de formalina a $10 \%$. Os atributos morfológicos selecionados foram: forma e posição da boca, distribuição e forma dos dentes, brânquias (primeiro arco direito) e estômago. Utilizouse de estereomicroscópio com câmara clara acoplada para ilustrar os aspectos morfológicos.

\section{RESULTADOS}

Foram coletados 169 exemplares de Trachelyopterus galeatus, dos quais 153 apresentaram conteúdo alimentar e foram avaliados, sendo 63 do reservatório de Santa Cruz e 90 de Umari. As análises dos itens permitiram a identificação de três grandes categorias alimentares: Animal, Vegetal e Detrito/ Sedimento (detrito é constituído predominantemente por material animal e vegetal em elevada taxa de decomposição, e o sedimento apresenta predomínio de material inorgânico - areia ou silte). Dentro da categoria animal os insetos constituíram maior diversidade, representados por diferentes ordens.

A maior ingestão de itens de origem animal, correu principalmente sob os insetos autóctones (aqueles que vivem pelos menos um estágio do ciclo de vida no ambiente aquático: Coleoptera, Odonata, Diptera, Ephemenoptera, Hemiptera, Trichoptera), camarão, molusco e peixe. Também foi registrado o consumo de insetos alóctones (aqueles que não são naturais do ambiente aquático, que foram levados para esse ambiente através da chuva, vento ou de alguma outra forma: Hymenoptera, Lepidoptera e Orthoptera) (Tab. I). Esses registros evidenciam o hábito carnívoro da espécie, consumindo insetos de origem alóctones e autóctones, mas com predomínio em ambos os reservatórios de molusco nos dois primeiros períodos (fevereiro e maio), sendo que noss dois últimos períodos (agosto e novembro), camarão foi o item mais consumido em Santa Cruz, e peixe e camarão em Umari, evidenciando a variação na alimentação de acordo com o período (Tab. I).

No reservatório de Santa Cruz, os itens Diptera, Trichoptera e Vegetal (sem origem determinada) estiveram presentes, ao contrário no reservatório de Umari, que não houve registro desses itens. Diptera apresentou relevância considerável na dieta da espécie no reservatório de Santa Cruz. Por outro lado, no reservatório de Umari os itens Hymenoptera, Ephemenoptera e Hemiptera estiveram presentes, porém esses itens não obtiveram grande relevância na dieta.

Os dois primeiros eixos da ordenação da NMDS evidenciaram que a espécie modificou sua dieta ao longo do tempo no reservatório de Santa Cruz (stress $=0,03$; PERMANOVA $F=3,07, p=0,0002$ ) (Fig. 3A), e o teste post-hoc da PERMANOVA indicou diferença entre os meses de fevereiro $v s$ agosto $(p=0,0004)$ e novembro $(p=0,02)$, além de maio vs agosto $(\mathrm{p}=0,0001)$ e novembro $(\mathrm{p}=0,03)$. Já no reservatório de Umari (Fig. 3B) a dieta da espécie também variou ao longo do tempo (stress $=0,25$; PERMANOVA F $=$ $2,09, \mathrm{p}=0,005$ ), e o teste de post-hoc evidenciou mudança no consumo dos recursos alimentares entre novembro $v s$ fevereiro $(p=0,02)$, maio $(p=0,003)$ e agosto $(p=0,03)$.

Com relação à avaliação espacial da dieta entre os dois reservatórios (Fig. 4) os dois primeiros eixos da ordenação

Tab. I. Índice Alimentar (IAi) com destaque (*) para os itens preferenciais e secundários, de acordo com a escala de RosecCHI \& NouAZE (1987), na dieta de Trachelyopterus galeatus (Linnaeus, 1766), nos reservatórios de Santa Cruz e Umari, Rio Grande do Norte, Brasil em fevereiro (Fev), maio (Mai), agosto (Ago) e novembro (Nov) de 2013 (1, itens autóctones; 2, itens alóctones; \#, item de origem não identificada).

\begin{tabular}{|c|c|c|c|c|c|c|c|c|c|}
\hline \multirow{2}{*}{ Categorias alimentares } & \multirow{2}{*}{ Itens } & \multicolumn{5}{|c|}{ Santa Cruz } & \multicolumn{3}{|c|}{ Umari } \\
\hline & & Fev & Mai & Ago & Nov & Fev & Mai & Ago & Nov \\
\hline \multirow{12}{*}{ Animal } & 1 Camarão & 4,91 & 13,28 & $* 87,22$ & $* 72,68$ & 13,89 & 16,32 & 10,81 & $* 83,46$ \\
\hline & 1 Peixe (escamas/espinhas) & 22,11 & & 0,60 & 1,16 & 0,37 & 0,1 & $* 72,14$ & 0,31 \\
\hline & 1 Molusco & $* 49,56$ & $* 64,43$ & 6,50 & 14,12 & $* 81,25$ & $* 76,45$ & 16,88 & 15,18 \\
\hline & 1 Coleoptera & 8,60 & 7,54 & 2,41 & 1,55 & 0,11 & 1,23 & 0,04 & \\
\hline & 2 Hymenoptera & & & & & 0,12 & 0 & & \\
\hline & 1 Odonata & & & 1,05 & 0,39 & 3,98 & 1,47 & 0,01 & 0,98 \\
\hline & 1 Diptera & 14,74 & 8,10 & & 0,24 & & & & \\
\hline & 1 Ephemenoptera & & & & & & 0,01 & & \\
\hline & 1 Hemiptera & & & & & & 0,35 & & \\
\hline & 1 Trichoptera & & & 2,01 & & & & & \\
\hline & 2 Lepidoptera & & 0,10 & & & & 0,67 & & \\
\hline & 2 Orthoptera & & & & 0,58 & & 0,1 & & \\
\hline Vegetal & \# Restos vegetais & 0,07 & & & 0,07 & & & & \\
\hline \multirow[t]{2}{*}{ Detrito/Sedimento } & 1 Detritos/sedimento & & 0,64 & 0,18 & & & 0,01 & & 0,02 \\
\hline & \# Material orgânico & & 5,91 & 0,02 & 9,18 & & 0,06 & & \\
\hline
\end{tabular}




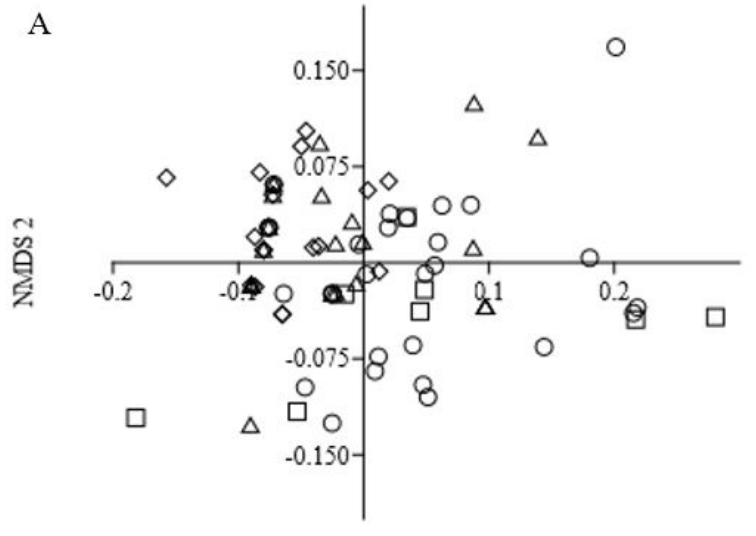

NMDS 1

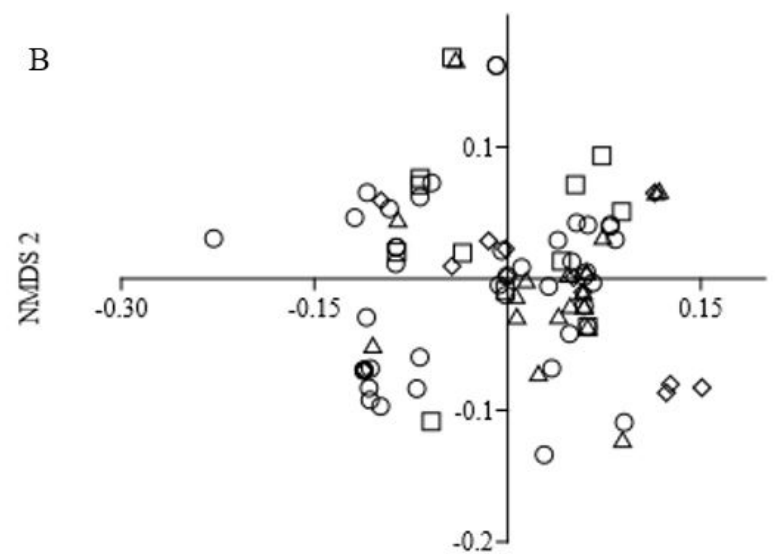

NMDS 1

Figura 3. Representação dos dois primeiros eixos da NMDS, mostrando a variação no consumo de recursos alimentares por Trachelyopterus galeatus (Linnaeus, 1766) nos reservatórios de Santa Cruz (A) (Eixo 1: 0,36 e Eixo 2: 0,22) e de Umari (B) (Eixo 1: 0,37 e Eixo 2: 0,30), Rio Grande do Norte, Brasil nos períodos de fevereiro $\square$, maio $\bigcirc$, agosto $\diamond$ e novembro $\triangle$ de 2013 .

demonstraram que a espécie apresenta dieta diferenciada entre os reservatórios estudados $($ stress $=0,28 ;$ PERMANOVA $F=$ $4,21 ; \mathrm{p}=0,0004)$. Apesar das mudanças significativas na dieta entre os reservatórios, T. galeatus manteve o mesmo padrão alimentar. Essa alteração é apontada pelo teste SIMPER, que demonstrou o uso diferencial dos recursos alimentares entre os reservatórios. Evidenciando os itens que apresentaram maior contribuição na dieta, que foram camarão $(33,01 \%)$ e molusco (32,13\%). Sendo que camarão apresentou uma maior abundância média $(0,43)$ no reservatório de Santa Cruz, por outro lado, molusco teve a maior abundância média $(0,52)$ no reservatório de Umari (Tab. II).

Quanto às características morfológicas, T. galeatus possui corpo cilíndrico e alongado com a cabeça levemente achatada dorso-ventralmente. A espécie possui boca terminal, porém ligeiramente superior, sendo considerada prognata. Ainda na cabeça há a presença de um par de barbilhões maxilares e dois pares de mentonianos (Fig. 5). Tanto a maxila superior quanto a inferior possuem dentículos curtos e pontiagudos agrupados em placas bem desenvolvidas, principalmente na região anterior. Imediatamente atrás da região das placas dentígeras, há botões gustativos reunidos (Fig. 6). A cavidade bucal, próximo à região faríngea, possui duas placas ósseas arredondadas, como duas almofadas, cobertas por dentículos cônicos (dentes faríngeos) (Fig. 6).

Há cinco arcos branquiais, o último menor e ligado à parede da cavidade branquial. Os rastros branquiais são curtos e de extremidade arredondada com espaço entre eles e com uma camada de muco recobrindo-os (Fig. 7). O estômago é ssaciforme, mas de paredes muito espessas e rígidas (Fig. 7), com ausência de cecos pilóricos.

\section{DISCUSSÃO}

O espectro alimentar de Trachelyopterus galeatus revelou uma dieta composta insetos de origem autóctone (Coleoptera, Odonata, Diptera, Ephemenoptera, Hemiptera,

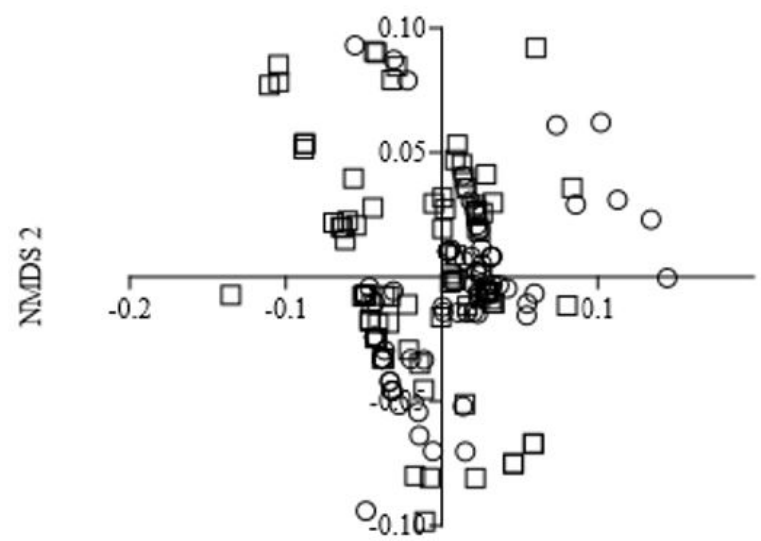

NMDS 1

Fig. 4. Representação dos dois primeiros eixos da NMDS (Eixo1: 0,30 e Eixo 2: 0,26$)$ demonstrando a variação no consumo de recursos alimentares por Trachelyopterus galeatus (Linnaeus, 1766) nos reservatórios de Santa Cruz $\bigcirc$ e de Umari $\square$, Rio Grande do Norte, Brasil nos períodos de fevereiro, maio, agosto e novembro de 2013.

Trichoptera) e insetos de origem alóctones (Hymenoptera, Lepidoptera e Orthoptera), além de camarão, molusco, peixe, bem como vegetal e material orgânico (sem origem definida). Contudo, a espécie variou o consumo dos itens ao longo do período estudado nos reservatórios de Santa Cruz e Umari, com predominância de camarão e moluscos. Entretanto, no reservatório de Umari o item peixe mostrou-se relevante na dieta da espécie, demonstrando, assim, uma adaptabilidade trófica entre os ambientes estudados. De acordo com a composição alimentar e prevalência dos itens camarão e molusco, a espécie foi classificação como carnívora.

A fração de fragmentos de vegetais encontrados nos conteúdos estomacais provavelmente ocorreu de forma acidental, algo comum em espécies que apresentam um caráter voraz que engole partes da vegetação que está por perto ao atacar sua presa (Moraes \& BARBOLA, 1995). 
Tab. II. Análise de SIMPER complementar a NMDS mostrando a contribuição e a abundância média dos itens alimentares para dissimilaridade da dieta de Trachelyopterus galeatus (Linnaeus, 1766) nos reservatórios de Santa Cruz (SC) e de Umari (UM), Rio Grande do Norte, Brasil em fevereiro, maio, agosto e novembro de 2013.

\begin{tabular}{|c|c|c|c|}
\hline Táxon & Contribuição \% & Abundância Média/SC & Abundância Média/UM \\
\hline Camarão & 33,01 & 0,43 & 0,36 \\
\hline Molusco & 32,13 & 0,33 & 0,53 \\
\hline Coleoptera & 7,43 & 0,08 & 0,02 \\
\hline Peixe & 6,90 & 0,05 & 0,12 \\
\hline Material orgânico & 5,25 & 0,09 & 0,002 \\
\hline Odonata & 4,54 & 0,04 & 0,04 \\
\hline Diptera & 3,92 & 0,07 & 0,000 \\
\hline Vegetal & 2,83 & 0,02 & 0,05 \\
\hline Hemiptera & 1,78 & 0,06 & 0,008 \\
\hline Orthoptera & 1,03 & 0,02 & 0,003 \\
\hline Lepidoptera & 0,69 & 0 & 0,009 \\
\hline Detritos e sedimento & 0,25 & 0,001 & 0,001 \\
\hline Ephemenoptera & 0,10 & 0 & 0,001 \\
\hline Trichoptera & 0,10 & 0,0009 & $5,49 \mathrm{E}-06$ \\
\hline Hymenoptera & 0,05 & 0 & 0,0003 \\
\hline
\end{tabular}
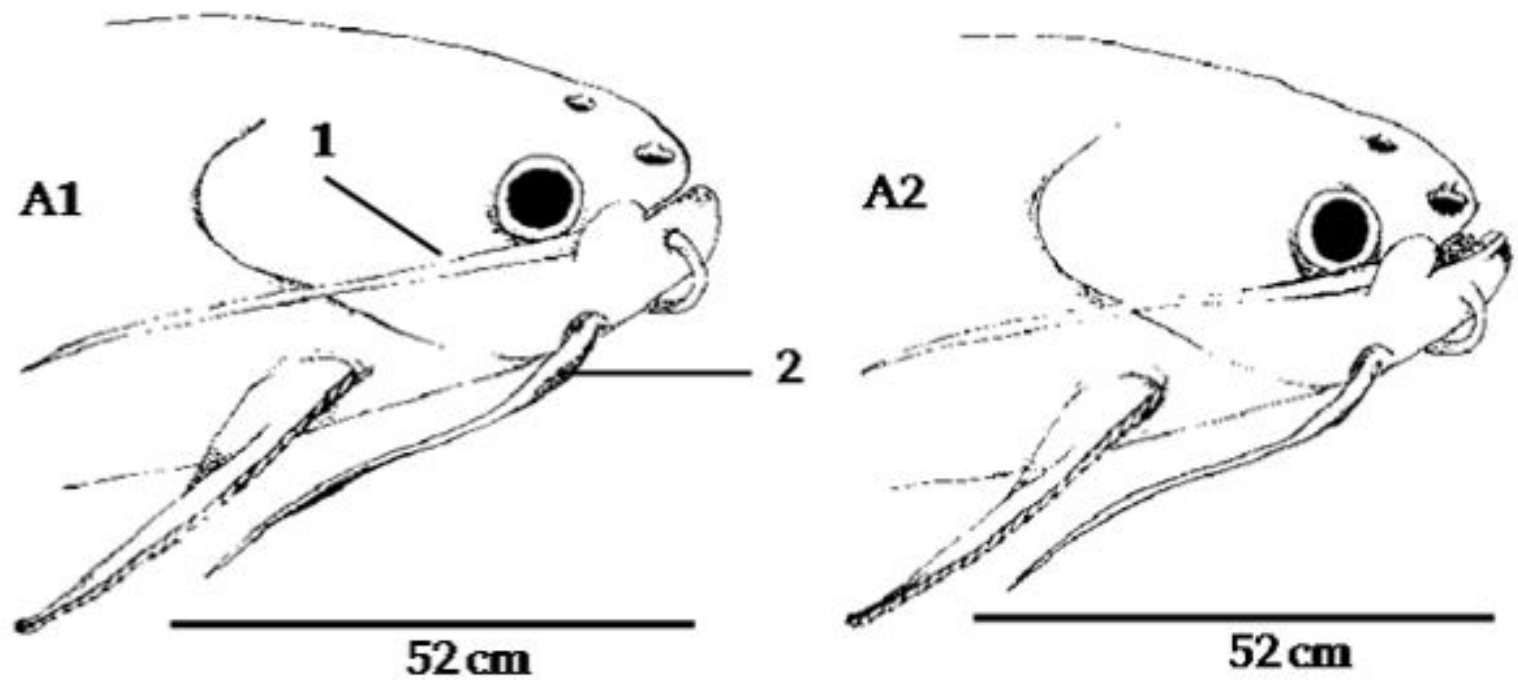

Fig. 5. Esquema da cabeça de Trachelyopterus galeatus (Linnaeus, 1766) com a boca fechada (A1) e aberta (A2) (1, barbilhão maxilar; 2, barbilhão mentoniano).

E também porque muitas espécies utilizam a estrutura física do ambiente como abrigo contra predadores, tais como vegetação, rocha, madeiras submersas como é o caso do camarão e peixes jovens e de pequeno porte (OLIVEIRA \& GOULART, 2000).

Estudos sobre a alimentação de $T$. galeatus em diferentes bacias hidrográficas (CLARO-JR et al., 2004; PERETTI \& ANDRIAN, 2004, 2008; XIMENES et al., 2011) classificam-na como onívora, com tendência à insetivoria. SANTOS (2005) classificou a espécie como onívora, mas com uma proporção equilibrada de itens de origem animal e vegetal em sua dieta, em trechos de dois rios na Bahia. Com relação ao estado do Rio Grande do Norte, estudos referentes à espécie são escassos, com destaque para os trabalhos realizados no rio Piranhas-Açu (Alto do Rodrigues, São Rafael e Açu), e açude
Corredor (Martins) que a classificaram como insetívora (GURGEL et al., 2002); a espécie também apresentou a dieta insetívora no estudo realizado no reservatório de Santa Cruz por Oliveira et al. (2016b). Nos reservatórios objetos deste estudo, embora exista a presença de insetos na dieta, estes não estiveram em proporções relevantes para se considerar a insetivoria como tendência.

A variação temporal no consumo dos itens alimentares por T. galeatus pode estar associada à pluviosidade da região. Os maiores valores registrados para moluscos ocorreram no período de maior precipitação (fevereiro e maio). Nesse período pode ocorrer a invasão da área marginal pelas águas e sucessão de plantas aquáticas (VASCONCELOS \& SÁ-OLIVEIRA, 2011). O aumento da área marginal disponibiliza novos habitats, itens alóctones e matéria orgânica para o sistema, que 


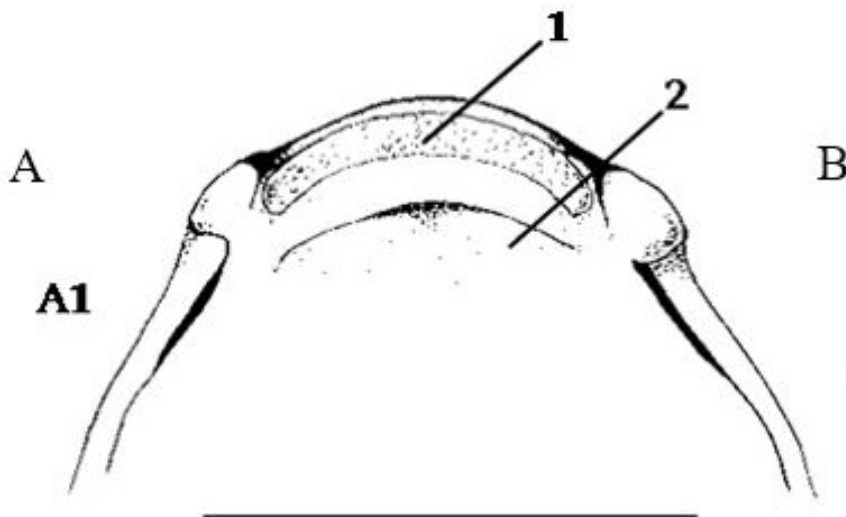

$3,6 \mathrm{~cm}$
B

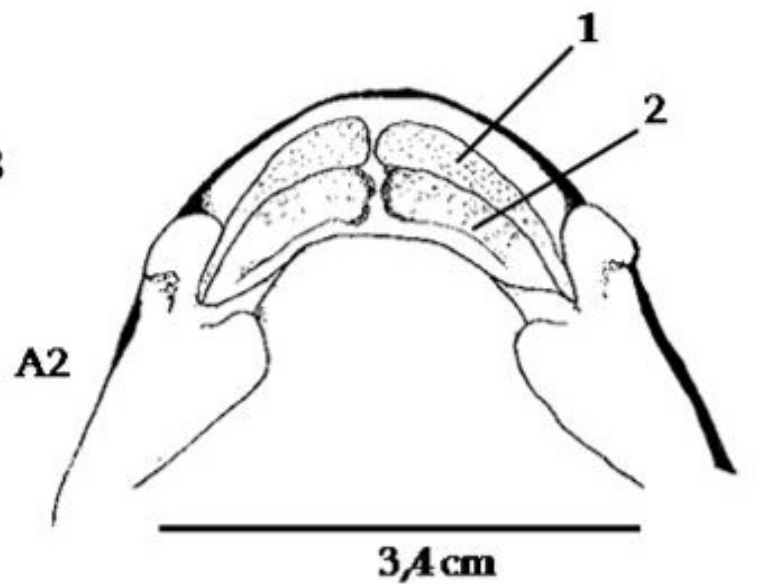

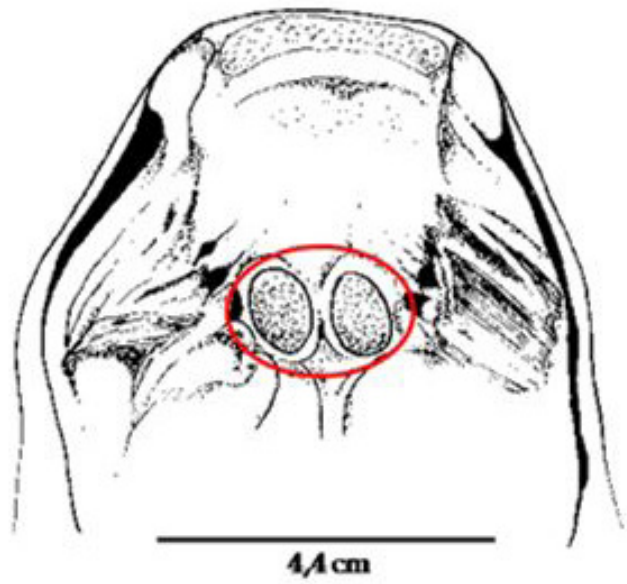

Fig. 6. Esquema da localização dos dentes de Trachelyopterus galeatus (Linnaeus, 1766) na maxila superior (A1) e na inferior (A2) (1, placa dentígera; 2, botões gustativos); localização das duas placas ósseas, contendo dentes faríngeos em destaque (círculo).

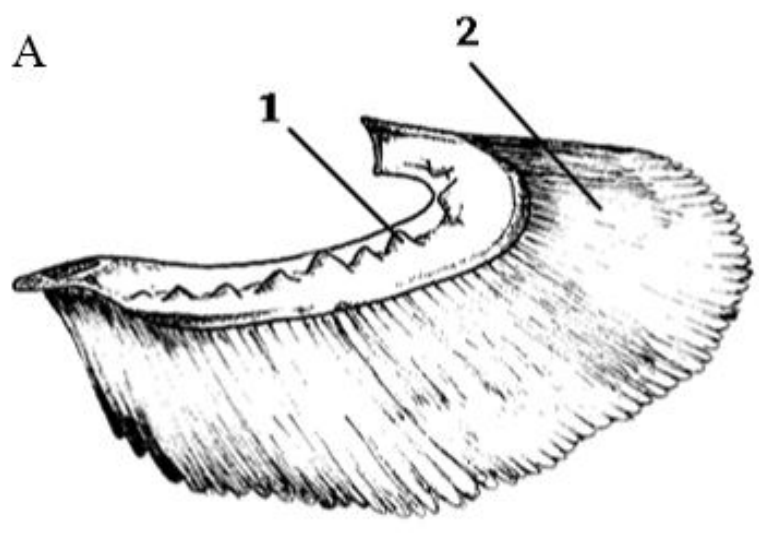

$2,3 \mathrm{~cm}$
B

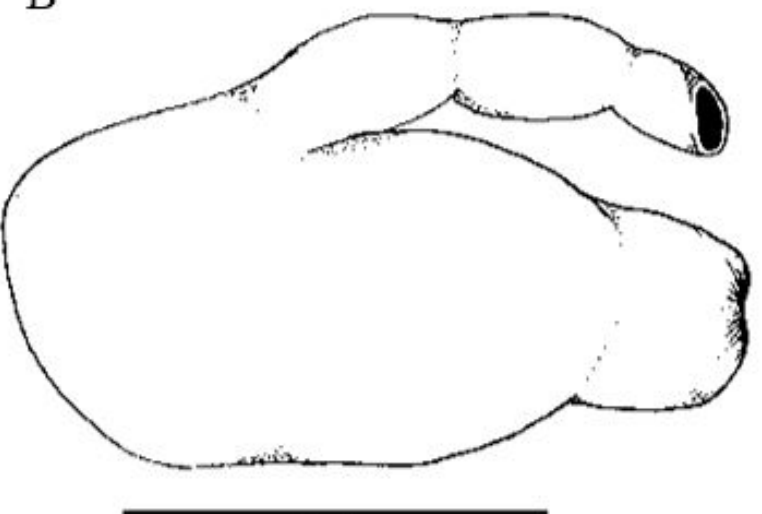

$2,4 \mathrm{~cm}$

Fig. 7. Esquema dos arcos branquiais de Trachelyopterus galeatus (Linnaeus, 1766) (A, rastros branquiais; 2, filamentos branquiais; B, estômago saciforme). 
servirá para os moluscos, bem como para outros organismos bentônicos como larvas de insetos, tornando-se esses recursos mais disponíveis na dieta de peixes (SILVA et al., 2012).

Moluscos são recursos alimentares de grande importância na dieta de peixes neotropicais, os quais podem ser tanto da ordem Gastropoda como Bivalve (WINEMILLER et al., 2008) as quais foram ordens que compuseram a dieta de T. galeatus. Com a inundação da vegetação marginal, além do aporte de material externo, ocorre o sombreamento de partes do corpo d'água, aumento na quantidade de material em suspensão, que diminui a penetração de luz, influenciando na transparência da água. O que diminui a intensidade luminosa e inibe o crescimento do fitoplâncton, tornando-se mais escasso para o camarão (BARBosa \& SOARES, 2009), diminuindo assim sua disponibilidade para T. galeatus. O aumento do nível de água do reservatório também fornece novos abrigos para o camarão dificultando a sua captura (FreIRE et al., 2012). Esses fatores justificam a predominância de moluscos no período de maior precipitação e camarão no período de menor precipitação.

As mudanças sazonais registradas demonstram que T. galeatus é uma espécie com uma dieta flexível, cujos hábitos alimentares e o tipo de alimento consumido dependem da abundância e da disponibilidade no ambiente (NUNN, 2012) geralmente facilitando sua predação. Seguindo a teoria do forrageamento ótimo (MACARTHUR \& PiANKA, 1966), em que os organismos são adaptados para obter alimento com o maior valor energético gastando o mínimo possível de energia, observou-se que a espécie também consumiu peixes, insetos, além de vegetais, detrito/sedimento e material orgânico. A maioria das espécies de peixes de água doce apresenta mudanças sazonais em suas dietas, decorrente das diversas táticas alimentares associadas à captura de itens, que contribui para o maior sucesso na predação e exploração dos recursos alimentares disponíveis no ambiente (ABELHA et al., 2001; Delariva et al., 2013). Essa estratégia é essencial, pois possibilita que as espécies possam persistir nos ambientes, uma vez que permitem alargar sua gama de recursos (Delariva et al., 2013). Para um peixe se especializar em determinado recurso, duas regras devem satisfazer esta condição: o peixe deve apresentar estrutura morfológica hábil para exploração do alimento e o recurso deve estar em quantidade suficiente, em biomassa, para suprir as necessidades do predador (LAGLER et al., 1977).

Com relação aos aspectos morfológicos, $T$. galeatus possui corpo cilíndrico e alongado e a cabeça levemente achatada dorso-ventralmente; boca terminal, porém ligeiramente superior, sendo considerada do tipo prognata. Ainda na cabeça há a presença de um par de barbilhões maxilares e dois pares de mentonianos. Essas características permitem que $T$. galeatus explore diferentes áreas, capturando alimento tanto na superfície quanto no fundo, não havendo uma preferência para explorar um determinado compartimento, resultando na sua diversificada composição alimentar (PERETTI \& ANDRIAN, 2008; SANTIN et al., 2015). No entanto, a dieta da espécie possivelmente foi influenciada principalmente pela largura relativa da boca e o comprimento relativo da cabeça, aspectos morfológicos que permite a espécie explorar diferentes recursos alimentares. FREITAS et al. (2017) evidenciaram que esses atributos ecomorfológicos em $A$. longimanus e $T$. galeatus permitem que as espécies se alimentem de itens maiores, como sementes e crustáceos aquáticos, respectivamente.

A morfologia dos rastros branquiais e a camada de muco observada recobrindo-os ajuda na apreensão de itens bentônicos, como larvas de insetos. O estômago saciforme com paredes rígidas e resistentes está em conformidade com a ampla gama de itens ingeridos, e explica a presença de itens de difícil digestão, tais como moluscos e coleópteros (Peretti \& Andrian, 2008), assim como, provavelmente, a carapaça quitinosa do camarão. De acordo com KJORSVIK et al. (2004), um sistema digestório eficiente capacita os indivíduos para a captura, a ingestão, a digestão e também a absorção do alimento. Os aspectos morfológicos descritos são semelhantes aos observados também em T. galeatus por Peretti \& ANDrian (2008) e SANTIN et al. (2015).

Segundo FERraris JR. (2003) os auquenipterídeos habitam nichos muito parecidos, são espécies noturnas, escondendo-se durante o dia em fendas de rocha e ramos e árvores submersas, o que provavelmente envolveu várias adaptações semelhantes em morfologia e comportamento (FrEITAs et al., 2017), sendo considerados generalizadas como carnívoras, predominando principalmente insetos, bem como o plâncton e peixes (FERRARIS JR., 2003). Esta aspecto explica a semelhança do hábito alimentar das espécies pertencente ao gênero Auchenipteridae, como insetívoros para Auchenipterus nuchalis (MÉRONA et al, 2008) insetívoros/ frugívoros para Auchenipterichthys longimanus (FREITAS et al., 2011) e Tatia intermedia (RAMíREZ et al., 2015) e Trachelyopterus galeatus (XIMENES et al., 2011) e insetívoros para Auchenipterichthys longimanus, Auchenipterus nuchalis, Trachelyopterus galeatus e Tatia intermedia (FREITAs et al., 2017).

No presente estudo $T$. galeatus também foi classificado como carnívoro, consumindo grande diversidade de insetos, mas com predominância dos itens molusco e camarão. Esse resultado pode está relacionado com a disponibilidade desses recursos alimentares nos locais onde a espécie foi estudada, confirmando seu caráter generalista. O registro dessa diversificada dieta, pode estar associada às mudanças ontogenéticas sofridas pela espécie ao longo de seu desenvolvimento, pois segundo SANTIN et al. (2015), estágios finais das larvas de T. galeatus consomem uma grande quantidade de presas diferentes, em decorrência das modificações morfológicas ocorridas durante os estágios larvais da espécie.

É comum, ao longo do desenvolvimento, larvas e juvenis de peixes incluírem em suas dietas presas maiores, bem como diversificar os itens consumidos (MAKRAKIs et al., 2005; NunN et al., 2007). Essas alterações frequentemente coincidem com a mudança no uso do habitat ou no comportamento ao longo do desenvolvimento (NuNN et al., 2012), além de estarem fortemente relacionadas às mudanças morfológicas (MAKRAKIS et al., 2005), permitindo ingerir, 
digerir e absorver os diferentes tipos de alimentos e explorar uma grande diversidade de itens disponíveis (SILVA et al., 2016), de acordo com morfologia do seu trato digestório.

Em ambos reservatórios Trachelyopterus galeatus apresentou dieta diversificada composta principalmente por insetos de origem alóctones e autóctones, moluscos e camarão; contudo, houve variação temporal entre os itens preferenciais na sua dieta. A variedade de itens alimentares foi maior no período chuvoso nos dois reservatórios, demonstrando a influência das chuvas na dieta da espécie. Quanto às características morfológicas, a posição ligeiramente superior e protrátil da boca, tubo digestório diferenciado (em estômago e intestino), além dos rastros branquiais, das placas de dentes e a presença de barbilhões, permitem que a espécie explore diferentes áreas do corpo hídrico, resultando em sua diversificada composição alimentar e, consequentemente, nas alterações registradas em sua dieta ao longo do período de estudo; neste sentido, as características morfológicas encontradas estão em conformidade ao consumo dos itens alimentares.

Agradecimentos. Ao Conselho Nacional de Desenvolvimento Científico e Tecnológico (CNPq) e a Fundação de Apoio a Pesquisa do Estado do Rio Grande do Norte (FAPERN) pelo apoio financeiro. À Universidade do Estado do Rio Grande do Norte (UERN), à Universidade Federal Rural do Semi-Árido (UFERSA) e ao Instituto Federal do Rio Grande do Norte (IFRN), campus de Apodi, pelo apoio logístico.

\section{REFERÊNCIAS BIBLIOGRÁFICAS}

Abelha, M. C. F.; Agostinho, A. A. \& Goulart, E. 2001. Plasticidade trófica em peixes de água doce. Acta Scientiarum, Biological Sciences 23(2):425-434.

ANA - AGÊNCIA NACIONAL DE ÁGUAS. 2007. Boletim de monitoramento dos reservatórios do nordeste do Brasil. Superintendência de Usos Múltiplos. Brasília, Agência Nacional de Águas. 23p.

Araújo, D. D. A.; Oliveira, J. F.; Costa, R. S. \& Novaes, J. L. C. 2016. Population structure and reproduction of a migratory fish Leporinus piau (Characiformes: Anostomidae) in the reservoir tropical semiarid. Revista de Biologia Tropical 64(4):1-13.

Barbosa, J. M. \& SoAres, E. C. 2009. Perfil da ictiofauna da bacia do São Francisco: estudo preliminar. Revista Brasileira de Engenharia de Pesca 4(1):155-172.

Bastos, R. F.; Miranda, S. F. \& Garcia, A. M. 2013. Diet and feeding strategy of Characidium rachovii (Characiformes, Crenuchidae) in coastal plain streams of southern Brazil. Iheringia, Série Zoologia 103(4):335-341.

Borges, S. A. G. V.; Gurgel, H. C. B. \& Canan, B. 1999. Estrutura populacional de Parauchenipterus galeatus Linnaeus, 1766 (Siluriformes, Auchenipteridae), da Lagoa de Jiqui, Parnamirim, Rio Grande do Norte. Revista CERES 46(264):209-218.

Claro-Jr, L.; Ferreira, E.; Zuanon, J. \& Araújo-Lima, C. 2004. $\mathrm{O}$ efeito da floresta alagada na alimentação de três espécies de peixes onívoros em lagos de várzea da Amazônia central, Brasil. Acta Amazônica 34(1):133-137.

Costa, M. R. A.; Attayde, J. L. \& Becker, V. 2016. Effects of water level reduction on the dynamics of phytoplankton functional groups in tropical semi-arid shallow lakes. Hydrobiologia 778(1):1-15.

Davies, P. M.; Bunn, S. E. \& Hamilton, S. K. 2008. Primary production in tropical streams and rivers. In: Dudgeon, D. ed. Tropical stream ecology. London, Academic Press, p. 23-42.

Delariva, R. L.; Hahn, N. S. \& Kashiwaqui, E. A. L. 2013. Diet and trophic structure of the fish fauna in a subtropical ecosystem: impoundment effects. Neotropical Ichthyology 11(4):891-904.

EMPARN - Empresa de Pesquisa Agropecuária do Rio Grande do Norte.
Disponível em < http://189.124.201.150/monitoramento/monitoramento. php $>$. Acessado em 01.09.2016.

Felipe, T. R. A. \& SuAREZ, Y. R. 2010. Caracterização e influência dos fatores ambientais nas assembleias de peixes de riachos em duas microbacias urbanas, Alto Rio Paraná. Biota Neotropica 10(2):143-151.

Ferraris JR., C. J. 2003. Family Auchenipteridae. In: ReIs, R. E.; Kullander, S. O. \& Ferraris Jr, C. J. eds. Check List of the Freshewater Fishes of South and Central America. Porto Alegre, Edipucrs, p. 473-485.

Freire, J. L.; Marques, C. B. \& Silva, B. B. 2012. Estrutura populacional e biologia reprodutiva do camarão-da-Amazônia Macrobrachium amazonicum (Heller,1862) (Decapoda: Palaemonidae) em um estuário da região nordeste do Pará, Brasil. Brazilian Journal of Aquatic Science and Technology 16(2):65-76.

Freitas, T. M. S.; Almeida, V. H. C.; Valente, R. M. \& Montag, L. F. A. 2011. Feeding ecology of Auchenipterichthys longimanus (Siluriformes: Auchenipteridae) in a riparian flooded forest of Eastern Amazonia, Brazil. Neotropical Ichthyology 9:629-636.

Freitas, T. M.; S.; Montag, L. F. \& Barthem, R. B. 2017. Distribution, feeding and ecomorphology of four species of Auchenipteridae (Teleostei: Siluriformes) in Eastern Amazonia, Brazil. Iheringia, Série Zoologia 107:e2017008.

Gurgel, H. C. B.; Lucas, F. D. \& Souza, L. L. G. 2002. Dieta de sete espécies de peixes do semi-árido do Rio Grande do Norte, Brasil. Revista de Ictiologia10(1/2):7-16.

HAHN, N. S. \& FuGI, R. 2007 Alimentação de peixes em reservatórios brasileiros: alterações e consequências nos estágios iniciais do represamento. Oecologia Brasiliensis 11(4):469-480.

Hammer, O. 2017. PAST- PAleontological STatistics, version 3.14. Disponível em $<$ http://http://folk.uio.no/ohammer/past/>.

Hellawell, J. M. \& ABel, R. A. 1971. A rapid volumetric method for the analysis of the food of fishes. Journal of Fish Biology 3(1):29-37.

Hynes, H. B. N. 1950. The food of freshwater sticklebacks (Gasterosteus aculeatus and Pygosteus pungitius) with a review of methods used in studies of the food of fishes. Journal of Animal Ecology 19(1):36-58.

HysLOP, E. J. 1980. Stomach contents analysis - a review of methods and their application. Journal Fish Biology 17(4):411-429.

KaWaKami, E. \& Vazzoler, G. 1980. Método gráfico e estimativa de índice alimentar aplicado no estudo de alimentação de peixes. Boletim do Instituto Oceanográfico 29(2):205-207.

Kuorsvik, E.; Pittman, K. \& Pavlov, D. 2004. From the fertilization to the end of metamorphosis - functional development. In: MoKsness, E.; KJorsvik, E. \& Olsen, Y. eds. Culture of coldwater marine fish. Oxford, Blackwell Science, p. 204-271.

Lagler, K. F.; Bardach, J. E.; Miller, R. R. \& Passino, D. R. 1977. Ichthyology. New York, John Wiley and Sons. 506p.

MacArthur, R. H. \& Pianka, E. R. 1966. On optimal use of a patchy environment. American Naturalist 100:603-609.

Makrakis, M. C.; Nakatani, K.; Bialetkzi, A.; Sanches, P. V.; Baumgartner, G. \& Gomes, L. C. 2005. Ontogenetic shifts in digestive tract morphology and diet of fish larvae of the Itaipu reservoir, Brazil. Environmental Biology Fishes 72(1):99-107.

MCCAFFERTY, W. P. 1981. Aquatic Entomology. Boston, Jones and Bartlett Publishers. 448p.

Medeiros, T. N.; Rocha, A. A.; Santos, N. C. \& Severi, W. 2014. Influence of the hydrological level on the diet of Leporinus reinhardtii (Characiformes, Anostomidae) in a semi-arid Brazilian reservoir. Iheringia, Série Zoologia 104(3):290-298.

Mérona, B. \& RANKIn-DE-MÉronA, J. 2004. Food resource partitioning in a fish community of the central Amazon floodplain. Neotropical Ichthyology 2(2):75-84.

Mérona B.; Hugueny, B.; Tejerina-Garro, F. L. \& Gautheret, E. 2008. Diet-morphology relationship in a fish assemblage from a mediumsized river of French Guiana: the effect of species taxonomic proximity. Aquatic Living Resources 21:171-184.

Merritt, R. W. \& Cummins, K. W. 1996. An introduction to the aquatic insects of North America. 3ed. Dubuque, Kendall/Hunt. 722p.

Moraes, M. F. P. G. \& Barbola, I. F. 1995. Hábito alimentar e morfologia do tubo digestivo de Hoplias malabaricus (Osteichthyes: Erythrinidae) da Lagoa Dourada, Ponta Grossa, Paraná, Brasil. Acta Biológica 24(1-4):1-23. 
Nakatani, K.; Agostinho, A. A.; Baumgartner, G.; Bialetzki, A.; Sanches, P. V.; Makrakis, M. C. \& Pavanelli, C. S. 2001. Ovos e larvas de peixes de água doce: desenvolvimento e manual de identificação. Maringá, EDUEM. 378p.

Needham, J. G. \& NeEdham, P. R. 1982. Guia para el estudio de los seres vivos de las aguas dulces. Barcelona, Reverté S. A. 131p.

Novakowski, G. C.; Hahn, N. S. \& Fugi, R. 2007. Alimentação de peixes piscívoros antes e após a formação do reservatório de Salto Caxias, Paraná, Brasil. Biota Neotropica 7(2):149-154.

Nunn, A. D.; Harvey, J. P. \& Cowx, I. G. 2007 The food and feeding relationships of larval and $0+$ year juvenile fishes in lowland rivers and connected waterbodies. I. Ontogenetic shifts and interspecific diet similarity. Journal of Fish Biology 70(3):726-742.

Nunn, A. D.; Tewson, L. H. \& Cowx, I. G. 2012. The foraging ecology of larval and juvenile fishes. Reviews in Fish Biology and Fisheries 22(2):377-408.

Oliveira, E. F. \& Goulart, E. 2000. Distribuição espacial de peixes em ambientes lênticos: interação de fatores. Acta Scientiarum, Biological Sciences 22(2):445-453.

Oliveira, J. F.; Costa, R. S.; Novaes, J. L. C.; Rebouças, L. G. F.; MoraisSegundo, A. L. N. \& Peretti, D. 2016a. Efeito da seca e da variação espacial na abundância de indivíduos nas guildas tróficas da ictiofauna em um reservatório no Semiárido Brasileiro. Boletim do Instituto de Pesca 42(1):51-64

Oliveira, J. F.; Morais-Segundo, A. L. N. M.; Novaes, J. L. C.; Costa, R. S.; FrançA, J. S. \& Peretti, D. 2016b. Estrutura trófica da ictiofauna em um reservatório do semiárido brasileiro. Iheringia, Série Zoologia 106:1-9.

Oliveira, M. C.; Silva Júnior, M. C. \& Ribeiro, J. F. 2011. Perturbações e invasões biológicas: ameaças para a biodiversidade nativa? CEPPG Revista (Catalão) 25(2):166-183

Peretti, D. \& Adrian, I. F. 2004. Trophic structure of fish assemblages in five permanent lagoons of the high Paraná River floodplain, Brazil. Environmental Biology of Fishes 71(1):95-103.

Peretti, D. \& Adrian, I. F. 2008. Feeding and Morphological analysis of the digestive tract of four species of fish (Astyanax altiparanae, Parauchenipterus galeatus, Serrasalmus marginatus and Hoplias aff. malabaricus) from the upper Paraná River floodplain, Brazil. Brazilian Journal of Biology 68(3):671-679.

Pessoa, E. K. R.; Silva, N. B.; Araujo, A. \& Chellappa, S. 2012. Morphohistology of the digestive tract of the carnivorous fish Hoplias malabaricus (Bloch, 1794) (Osteichthyes: Erythrinidae). Animal Biology Journal 3(4):145-158.

Pinto, G. A.; Rocha, A. A. F. \& Lacerda, N. C. 2011. Variação sazonal na dieta de Triportheus guentheri (Garman, 1890) (Actinopterygii, Characidae), no reservatório de Sobradinho, rio São Francisco, BA. Boletim do Instituto de Pesca 37(3):295-306.

Ramírez, F.; DaVEnPort, T. L. \& MoJiCA, J. I. 2015. Dietary-morphological relationships of nineteen fish species from an Amazonian terra firme blackwater stream in Colombia. Limnologica 52:89-102.

RosecchI, E. \& NouAze, Y. 1987. Comparaison de cinq indices alimentaires utilisés dans l'analyse des contenus stomacaux. Revue des Travaux de l'Institut des Pêches Maritimes 49(3-4):111-123.

Santin, M. T. M.; Baggio, M. M.; Agostinho, A. A. \& BialetzKi, A. 2015. Mudanças ontogênicas no trato digestório e na dieta de Trachelyopterus galeatus. Boletim do Instituto de Pesca 41(1):57-68.

SAntos, A. C. A. 2005. Ecologia alimentar do molé, Trachelyopterus galeatus Linnaeus, 1766 (Siluriformes, Auchenipteridae), em trechos inferiores dos rios Santo Antônio e São José (Chapada Diamantina, Bahia). Sitientibus, Série Ciências Biológicas 5:93-98.

SEMARH - Secretaria de Recursos Hídricos e Meio Ambiente do Estado do Rio Grande do Norte. Disponível em < http://www.semarh.rn.gov. $\mathrm{br} />$. Acessado em 20.06.2016.

Silva, C. M.; Honorato, E. V.; Silva Filho, C. A. \& Silveira, P. B. 2016. $40 \mathrm{~K}$ como bioindicador de poluição do rio Capibaribe em Recife-PE. HOLOS 32(4):67-76.

Silva, D. A.; Pessoa, E. K. R.; Costa, S. A. G. L.; Chellappa, N. T. \& Chellappa, S. 2012. Ecologia alimentar de Astyanax lacustris (Osteichthyes: Characidae) na Lagoa do Piató, Assú, Rio Grande do Norte, Brasil. Biota Amazônia 2(1):74-82.

Sotres, M. G. M.; Menezes, N. A. \& Junk, W. J. 2006. Adaptations of fish species to oxygen depletion in a central Amazonian floodplain lake. Hydrobiologia 568(1):353-367.

Vasconcelos, H. C. G. \& SÁ-Oliveira, J. C. 2011. Alimentação de Potamotrygon motoro (Chondrichthyes, Potamotrygonidae) na planície de inundação da APA do Rio Curiaú, Macapá-Amapá-Brasil. Biota Amazônia 1(2):66-73.

VAzzoler, A. E. A. M. 1996. Biologia da reprodução de peixes teleósteos: teoria e prática. Maringá, Universidade Estadual de Maringá. 169p.

Winemiller, K. O.; Agostinho, A. A. \& Caramachi, E. P. 2008. Fish Ecology in Tropical Streams. In: DudgeOn, D. ed. Tropical Stream Ecology. Amsterdam, Elsevier, p. 107-146.

Ximenes, L. Q. L.; Mateus, L. A. D. F. \& Penha, J. M. F. 2011. Variação temporal e espacial na composição de guildas alimentares da ictiofauna em lagoas marginais do Rio Cuiabá, Pantanal Norte. Biota Neotropica 11(1):205-215.

ZAVALA-CAMIN, L. A. 1996. Introdução aos estudos sobre alimentação natural em peixes. Maringá, EDUEM. 129p. 